\title{
User Preferences on Route Instruction Types for Mobile Indoor Route Guidance
}

\author{
Laure De Cock* ${ }^{\mathbb{D}}$, Kristien Ooms $\mathbb{D}^{D}$, Nico Van de Weghe $\mathbb{D}$, Nina Vanhaeren $\mathbb{D}^{\mathbb{D}}$ and \\ Philippe De Maeyer
}

Department of Geography, Ghent University, B-9000 Ghent, Belgium; kristien.ooms@ugent.be (K.O.); nico.vandeweghe@ugent.be (N.V.d.W.); nina.vanhaeren@ugent.be (N.V.); philippe.demaeyer@ugent.be (P.D.M.)

* Correspondence: laudcock.decock@ugent.be

Received: 8 September 2019; Accepted: 21 October 2019; Published: 25 October 2019

\begin{abstract}
Adaptive mobile wayfinding systems are being developed to ease wayfinding in the indoor environment. They present wayfinding information to the user, which is adapted to the context. Wayfinding information can be communicated by using different types of route instructions, such as text, photos, videos, symbols or a combination thereof. The need for a different type of route instruction may vary at decision points, for example because of its complexity. Furthermore, these needs may be different for different user characteristics (e.g., age, gender, level of education). To determine this need for information, an online survey has been executed where participants rated 10 different route instruction types at several decision points in a case study building. Results show that the types with additional text were preferred over those without text. The photo instructions, combined with text, generally received the highest ratings, especially from first-time visitors. 3D simulations were appreciated at complex decision points and by younger people. When text (with symbols) is considered as a route instruction type, it is best used for the start or end instruction.
\end{abstract}

Keywords: indoor route guidance; route instruction types; online survey; decision point characteristics; user characteristics

\section{Introduction}

Wayfinding in an indoor environment can be challenging. To assist people in their wayfinding decisions, location based systems (LBS) are being developed, which can provide wayfinding information on a mobile screen based on the user's location [1]. This wayfinding information can be conveyed to the user in two ways: an overview map or turn-by-turn instructions. A disadvantage of overview maps is the difficulty to visualize them on mobile devices because of the limited screen size [2]. Additionally, they often show unnecessary information for the navigation task at hand and a mental rotation can be required to align the map with the navigator's viewpoint [3,4]. An advantage of overview maps is that they enable wayfinders to improve their cognitive map of the place, although they often prefer to reach their destination quickly [5,6]. The second way of conveying wayfinding information is by giving turn-by-turn instructions to the user at every decision point [7]. This way, the system demands a lot of attention of the user (i.e., at every decision point), but it can also reduce uncertainty at decision points. However, only good wayfinding instructions will reduce uncertainty, whereas bad instructions can confuse the wayfinder and increase cognitive load [8]. This rise in cognitive load is caused by giving either insufficient information or extensive information [9]. To avoid the generation of confusing route instructions, the amount and type of wayfinding information that is needed at every decision point has to be determined. This need for information depends on the context, which can be defined as every factor that influences the interaction between user and application. At every new decision point, the context is different so a user's needs will change as well. Therefore, adaptive systems are being 
developed, which automatically adapt their characteristics to the context [1]. By adapting the given route information to the context, the route instructions will adhere better to the wayfinder's mental representation of the route and will therefore also demand less attention of the user $[10,11]$.

One of the elements that can be adapted by an adaptive system is the type of turn-by-turn instruction. Using a certain type at a certain decision point can improve wayfinding performance because every type has specific characteristics which may be better suited for certain decision points [2]. Five types of turn-by-turn instructions can be distinguished: symbols, text, photos, 3D simulations and maps. The first type has the advantage of being highly abstract, so the processing of the symbol induces a low cognitive load on the users. However, this abstraction of information can also be a disadvantage when more details are necessary to make a wayfinding decision [12]. When the symbol cannot be easily translated to the environment, a high cognitive load will be induced. The same contrast between abstraction and a more elaborate description needs to be considered when textual instructions are used. A long and elaborate instruction may be needed at open spaces, which are numerous indoors. Salient landmarks on the other hand are limited indoors, while good route instructions should refer to these elements [13]. Advantages of textual instructions are the limited resources needed for their generation and the ease of combining them with other instruction types [12]. Subsequently, several researchers have investigated the use of photos and 3D simulations for indoor wayfinding support. These types provide the user with a large amount of information in a short amount of time [14]. The realistic visualization makes it easier to recognize landmarks, but the interpretation of the extensive amount of information can imply a high cognitive load [15]. Photos need to be collected in the building at every decision point, so this type of route instruction demands more resources than text and symbols. 3D simulations demand even more resources, although the building information model (BIM) can be used to calculate routes and extract 3D simulations, if they are available [16]. Finally, maps are the most common type to visualize outdoor route instructions. However, switching between the egocentric perspective of the user and the geocentric perspective of the map (even when the map is forward-up) can demand cognitive load [12]. Some authors therefore recommend to simplify the map views for navigation purposes (e.g., [17]). Additionally, map instructions can be easily obtained from the floorplans of buildings, but should be extended with materials and colors to improve the consistency with the real environment [4]. When materials, colors and landmarks are added to the maps, more resources will be necessary for the generation of the instructions [14].

Several researchers have compared route instruction types for wayfinding support, by conducting experiments with a user-centered design [18]. In this type of experiment, the end users of a system are involved in the early stages of development, which improves the usability [19]. Kray et al. (2003) [20] concluded from an indoor wayfinding experiment that most participants were faster with maps, although they indicated to like the 3D visualization more. Chittaro \& Burigat (2005) [12] found that participants felt more disoriented with maps than with photographs. This conclusion was also drawn by Walther-Franks \& Malaka (2008) [21]. However, a lot of studies on adaptive wayfinding aids focus on outdoor use with a vehicle. The findings in this domain should not be blindly accepted for pedestrian indoor wayfinding as both the environment and the users fundamentally differ. For example, pedestrians have to use objects like stairs and doors and are not bound to the road [15]. Additionally, most previous studies do not consider adapting the type of route instruction to the decision point. This adaptation could facilitate efficient and effective wayfinding because not all decision points require the same attention of the wayfinder [10]. As Giannopoulos et al. (2014) [8] state, to analyze the complexity of a wayfinding situation, three elements should be taken into account: the environment, the user and the instruction. The combination of these three factors gives a better idea of the complexity at a decision point than only one or two of them.

Therefore, an explorative study has been conducted to determine which context characteristics have an influence on route instruction type preference. An online survey has been executed where participants were guided along several routes with different types of route instructions, which were rated at every decision point. First, the ratings of all instruction types were compared to determine 
which types were preferred by the participants. Next, every instruction type was analyzed separately to determine which decision point categories and user characteristics had an influence on the ratings. By doing so, this explorative research aims to address the following research questions: which route instruction types are preferred by participants? Does the required action at a decision point, such as starting the route or changing levels, influence this preference? Do user characteristics, such as age and experience with wayfinding aids, have an influence on the preference? The answers to these questions can be used to develop an adaptive indoor wayfinding system that adapts the type of route instruction to the environment and the user.

This paper is organized as follows: in the next section, the case study building, the route instruction types, the online survey and the statistical analysis are discussed. Section 3 presents the results of the analysis. The discussion in Section 4 is structured per instruction type, and is followed by a conclusion in Section 5.

\section{Materials and Methods}

\subsection{Case Study Building}

The case study building of the online survey is a smart office building of Ghent University: the iGent tower. In the top three floors of the tower a location based system (LBS) with ultra-wideband (UWB) sensors is installed. Based on this study, a wayfinding system will be developed for this LBS that automatically gives turn-by-turn instructions to the users, adapted to their location. The layout of the iGent is similar to many office buildings: a central corridor with offices on the outside and staircases and an elevator on the inside (Figure 1). The corridor counts four decision points to make turns (1-4) and three decision points to change levels (5-7). The ninth and tenth floor are connected with a spiral staircase, which is decision point 8 . The eleventh floor lacks a spiral staircase and counts therefore only seven decision points.
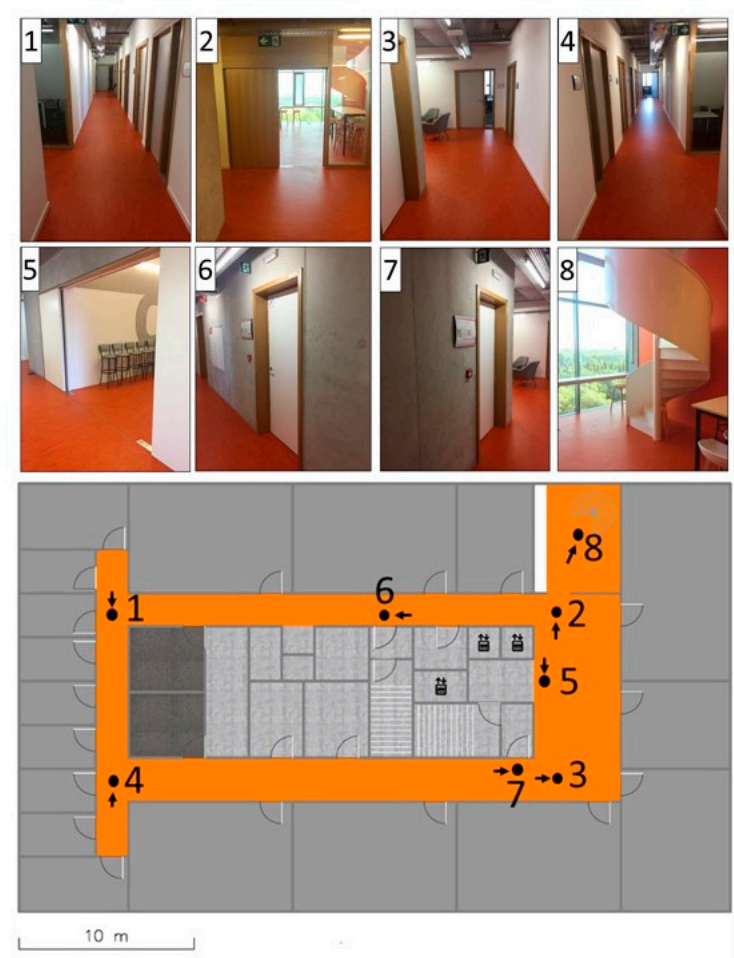

Figure 1. Floorplan of the iGent tower (ninth floor) with eight decision points. From every decision point an image is included, taken in the direction of the arrow. 
In Figure 2 an example of one of the routes in the study is shown with the text instructions. All routes are similar, but cover a different part of the case study building.

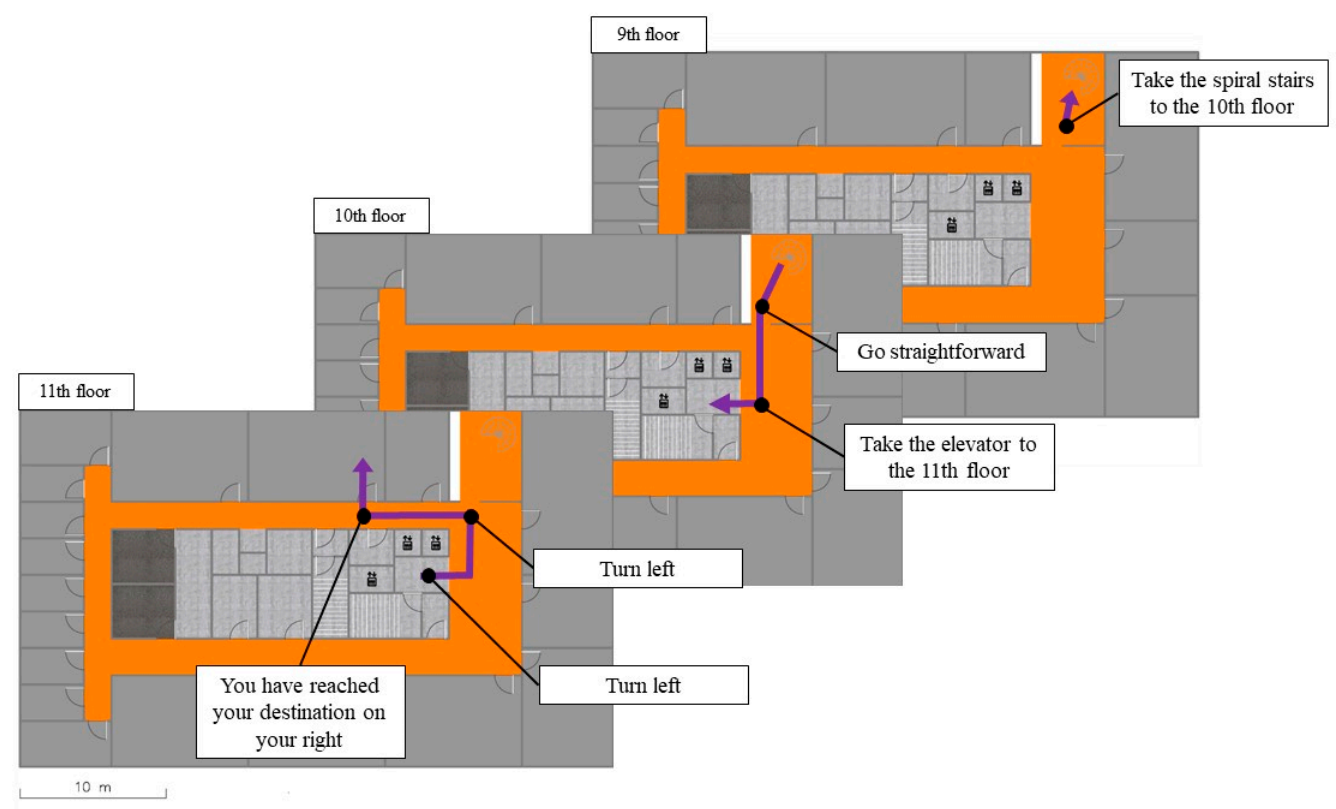

Figure 2. Example route.

\subsection{Route Instruction Types}

For every possible action at every decision point, ten types of route instructions were designed:

- $\operatorname{Map}(\mathrm{M})$

- $\quad$ Map + text (MT)

- $\quad$ Photo $(\mathrm{P})$

- $\quad$ Photo + text $(\mathrm{PT})$

- $\quad$ Text (T)

- $\quad$ Symbol + text (ST)

- $\quad 3$ D simulation - agent view (A)

- $3 \mathrm{D}$ simulation - agent view + text (AT)

- 3D simulation - overview $(\mathrm{O})$

- $3 \mathrm{D}$ simulation - overview + text $(\mathrm{OT})$

In Figure 3 an example of all types is shown. The A, O, M and P types were additionally combined with the text type by placing the text below the images or 3D simulations, resulting in four new instruction types according to the guidelines of Giannopoulos et al. (2014) [8]: MT, PT, AT and OT. The map instruction types were deduced from the floorplan of the building, but enhanced with realistic colors and materials as suggested by Puikkonen et al. (2009) [4]. Additionally, the suggestions of Ohm et al. (2017) were followed as well, by keeping the maps simple, in order not to confuse the wayfinder [17]. Symbols were added to both the map and photo type to visualize the required action. The 3D simulated route instructions were obtained by integrating the BIM of the building in the game engine Unity (https://unity.com/). With this open source software, videos of automatic navigation simulations were created. The difference between the agent type and the overview type is that the first is a video of the instruction being executed from the perspective of the wayfinder and the second is a static view of a dummy person executing the instruction. In this research, turn-by-turn instructions are given at the right time and place in the building. This way of conveying wayfinding information has been chosen because the turn-by-turn instructions will be used in an LBS with UWB sensors, installed in the building [22]. 

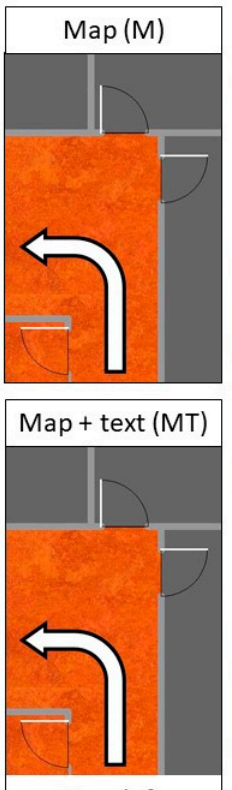

Turn left
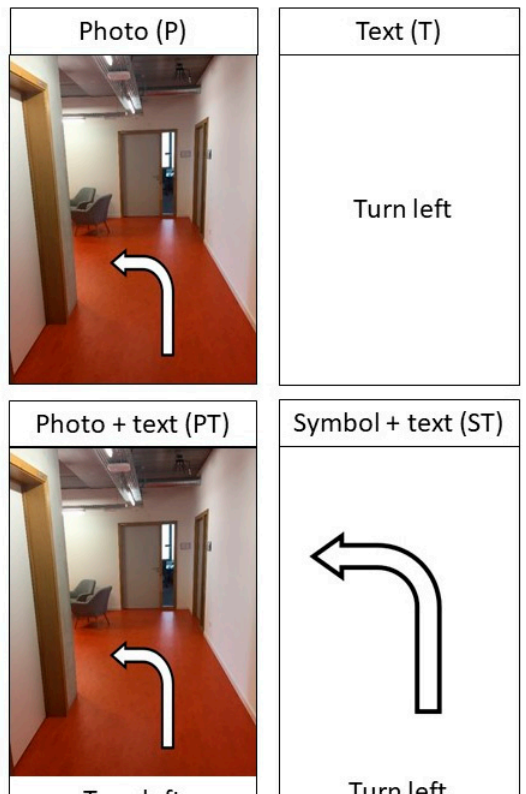

Turn left

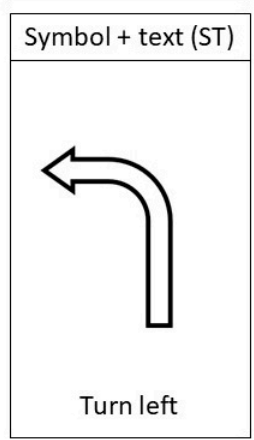

Figure 3. Route instruction types to turn left on decision point 3.

\subsection{Online Survey}

The online survey was published on the Amazon Mechanical Turk platform (https://www.mturk.com) and spread through several channels (e-mail, social media, flyers, etc.). To start the test, participants had to fill in their age, gender, nationality and educational level, and answer the following four general questions with 'yes', 'no' or 'I don't know':

- Do you easily get lost in buildings?

- Have you been to the iGent tower in Ghent, Belgium?

- Do you often use navigation aids to find your way indoors?

- Do you often use navigation aids to find your way outdoors?

Ten routes were recorded on video in the iGent tower. At every decision point along the routes (52 in total), the route video paused and a route instruction was shown. Participants had to indicate how good they found that instruction type for that decision point on a seven-grade Likert scale, after which the route video continued to the next decision point (Figure 4). If desired, additional remarks could be made at every decision point. For every route, a different instruction type was shown. Both the order of the routes and the order of the instruction types was randomized, thus all participants saw 10 instruction types and 10 routes, but not necessarily in the same combination. For every rating, the instruction type is stored together with the decision point and user characteristics of the participant.

\subsection{Statistical Analyses}

In a first step, the general preference for the instruction types was analyzed. Pairwise, one-tailed Mann-Whitney U tests (with Bonferroni correction) were executed to determine - in general - which instruction types were rated higher and which were rated lower. In a second step, each instruction type was individually analyzed, to determine which decision point characteristics on the one hand and user characteristics on the other hand had an influence on the ratings. To this end, 10 subdatasets were first created: one for every instruction type. Next, one-tailed pairwise Mann-Whitney U tests (with Bonferroni correction) were executed for every subdataset to determine if the instruction type received significantly different ratings at certain decision points or from certain user groups. 

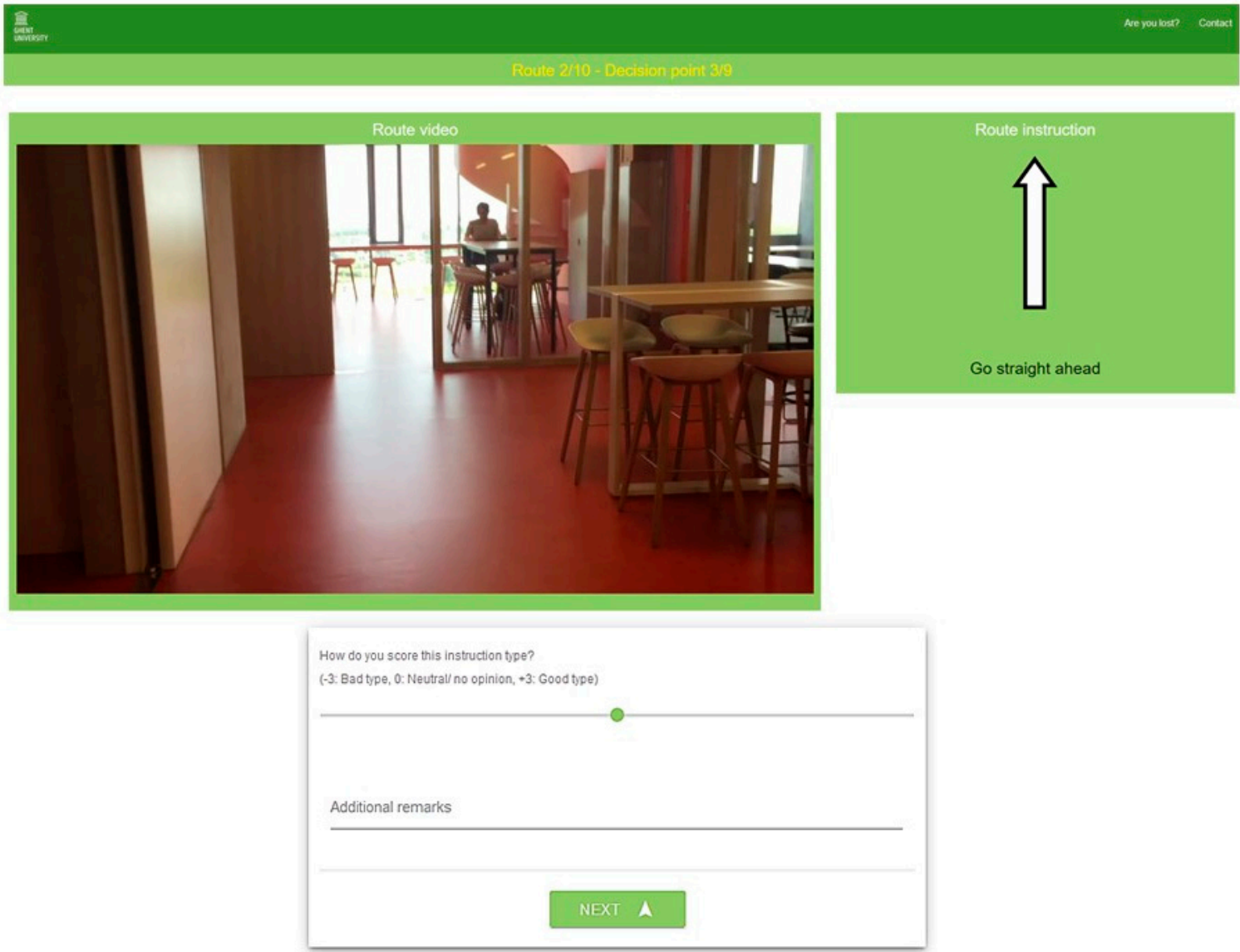

Figure 4. Sample question of the online survey with instruction type symbol + text (ST).

\section{Results}

\subsection{Participants}

In total, 240 participants completed the test of which 61 through the Amazon Mechanical Turk platform. Most participants were Belgian (103), but also people from the USA (35), India (28), Denmark (11) and 31 other countries took part. An overview of the user characteristics and the answers to the general questions can be found in Table 1.

Table 1. User characteristics.

\begin{tabular}{|c|c|c|c|c|}
\hline \multicolumn{5}{|c|}{ Age Class } \\
\hline \multirow{3}{*}{\multicolumn{3}{|c|}{$\begin{array}{c}1990-2000 \text { (young) } \\
\text { 1970-1990 (middle) } \\
\text { 1940-1970 (old) }\end{array}$}} & \multicolumn{2}{|c|}{$38 \%$} \\
\hline & & & \multicolumn{2}{|c|}{$46 \%$} \\
\hline & & & \multicolumn{2}{|c|}{$16 \%$} \\
\hline \multicolumn{5}{|c|}{ General questions } \\
\hline & (Q1) & (Q2) & (Q3) & (Q4) \\
\hline Yes $(y)$ & $33 \%$ & $9 \%$ & $25 \%$ & $80 \%$ \\
\hline No $(n)$ & $57 \%$ & $88 \%$ & $70 \%$ & $18 \%$ \\
\hline I don't know $(x)$ & $10 \%$ & $3 \%$ & $5 \%$ & $2 \%$ \\
\hline
\end{tabular}

(Q1) Do you easily get lost in buildings? (Q2) Have you been to the iGent tower in Ghent, Belgium? (Q3) Do you often use navigation aids to find your way indoors? (Q4) Do you often use navigation aids to find your way outdoors? 


\subsection{Descriptives}

The highest rating (+3) was most used and the lowest rating (-3) least used (Figure 5). Due to the skewness of this distribution, the median is not the symmetrical center of all values. Therefore, differences between groups were established with a Mann-Whitney U test, which tests whether the scores of two independent groups have a similar ranked distribution [23].

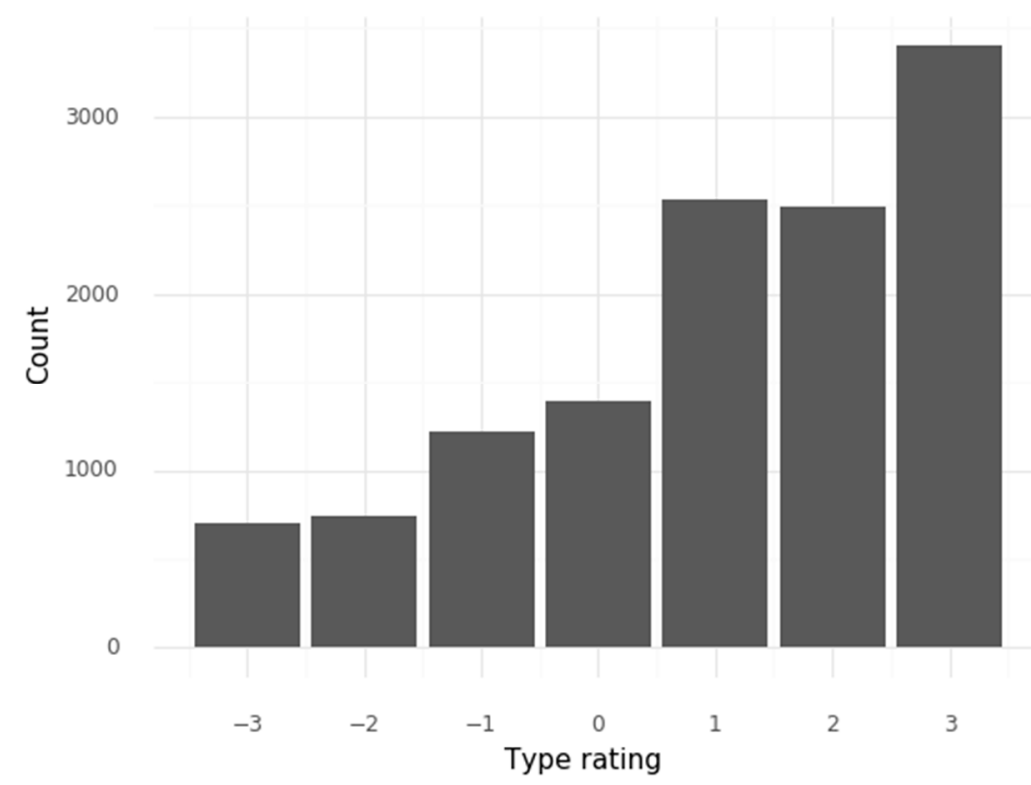

Figure 5. Histogram of all type ratings, based on a seven-grade rating scale: -3 (bad type) - +3 (good type).

Some trends in the data can already be observed from the medians (Table 2). All types without text, except for the picture type (P), have a lower median. Additionally, symbol and text (T, ST) got lower ratings as well. The mode values show that map, text and 3D-simualtion overview $(M, T, A)$ mostly got rated +1 , opposed to +3 for the other types.

Table 2. Descriptive statistics, based on a seven-grade rating scale: -3 (bad type)—+3 (good type).

\begin{tabular}{cccc}
\hline Type & IQR & Mode & Median \\
\hline A & 3 & 1 & 1 \\
AT & 3 & 3 & 2 \\
M & 3 & 1 & 1 \\
MT & 3 & 3 & 2 \\
O & 4 & 3 & 1 \\
OT & 3 & 3 & 2 \\
P & 2 & 3 & 2 \\
PT & 2 & 3 & 2 \\
ST & 3 & 3 & 1 \\
T & 3 & 1 & 1 \\
\hline
\end{tabular}

\subsection{Instruction Type Ratings}

The results of the pairwise Mann-Whitney $U$ tests can be seen in Table 3. The significance values are listed for the one-tailed tests that the types in the columns are higher rated than the types in the rows. 
Table 3. One-tailed significance values resulting from pairwise Mann-Whitney U tests (with Bonferroni correction) on the difference between instruction type ratings.

\begin{tabular}{|c|c|c|c|c|c|c|c|c|c|c|}
\hline$<$ & A & AT & $\mathbf{M}$ & MT & O & OT & $\mathbf{P}$ & PT & ST & $\mathrm{T}$ \\
\hline $\mathbf{A}$ & - & 0.00 * & 0.15 & 0.00 * & 0.01 * & 0.00 * & 0.00 * & 0.00 * & 0.99 & 0.99 \\
\hline AT & 0.99 & - & 0.99 & 0.99 & 0.99 & 0.99 & $0.02 *$ & 0.00 * & 0.99 & 0.99 \\
\hline $\mathbf{M}$ & 0.99 & 0.00 * & - & $0.00^{*}$ & 0.99 & 0.00 * & 0.00 * & $0.00 *$ & 0.99 & 0.99 \\
\hline MT & 0.99 & 0.99 & 0.99 & - & 0.99 & 0.99 & 0.99 & $0.00 *$ & 0.99 & 0.99 \\
\hline O & 0.99 & 0.00 * & 0.99 & 0.00 * & - & 0.00 * & 0.00 * & 0.00 * & 0.99 & 0.99 \\
\hline OT & 0.99 & 0.99 & 0.99 & 0.99 & 0.99 & - & 0.19 & $0.00 *$ & 0.99 & 0.99 \\
\hline $\mathbf{P}$ & 0.99 & 0.99 & 0.99 & 0.99 & 0.99 & 0.99 & - & 0.00 * & 0.99 & 0.99 \\
\hline PT & 0.99 & 0.99 & 0.99 & 0.99 & 0.99 & 0.99 & 0.99 & - & 0.99 & 0.99 \\
\hline $\mathrm{ST}$ & 0.99 & 0.00 * & 0.99 & $0.00^{*}$ & 0.99 & 0.00 * & 0.00 * & 0.00 * & - & 0.99 \\
\hline$T$ & 0.86 & 0.00 * & 0.00 * & 0.00 * & 0.00 * & 0.00 * & 0.00 * & 0.00 * & 0.01 * & - \\
\hline
\end{tabular}

Results show that instruction types with additional text (AT, MT, OT, PT) received higher scores than the same types without text (A, M, O, P). This preference for combined instruction types could also be concluded from the median values of the ratings (Table 2). The photo types (PT, P) received the highest ratings; followed by the 3D and map types with text (AT, MT, OT); next, the 3D and map types without text and symbols (A, M, O, ST); and finally, the text type (T). Furthermore, it can be noticed that $3 \mathrm{D}$ instructions from overview perspective $(\mathrm{O})$ were preferred over $3 \mathrm{D}$ instructions from the wayfinder's perspective (A).

\subsection{Context}

With this method, every instruction type was analyzed separately. First the difference in ratings between decision points was determined for every instruction type, next the difference in ratings between user characteristics for every instruction type. This results in a ranking of context (i.e., user and decision point) characteristics for every instruction type. Only the differences that are statistically significant at the $95 \%$ confidence level are included in the results.

\subsubsection{Ranking of Decision Point Characteristics}

First, all decision points of the case study building (Figure 1) were categorized in two different ways: a general classification and a more elaborate one where the level and turn categories are subdivided in three and two subclasses (Table 4). The classification of decision points is based on the action of the route instruction at that point.

Table 4. Categorization of decision points.

\begin{tabular}{|c|c|c|}
\hline & Description & Decision Points on Figure 1 \\
\hline Level $^{\text {a }}$ & $\begin{array}{l}\text { Decision points where one can change floors } \\
\text { (e.g., stairs or elevators). }\end{array}$ & $5,6,7,8$ \\
\hline Elevator ${ }^{b}$ & Decision point where one can change floors with an elevator. & 5 \\
\hline Stairs ${ }^{b}$ & Decision points where one can change floors with the stairs. & 6,7 \\
\hline Spiralstairs ${ }^{\mathrm{b}}$ & $\begin{array}{c}\text { Decision point in the kitchen where one can change floors with } \\
\text { the spiral stairs. }\end{array}$ & 8 \\
\hline Turn $^{a}$ & $\begin{array}{l}\text { Decision points where only turns can be made (e.g., turn left, } \\
\text { turn right, go straightforward). }\end{array}$ & $1,2,3,4$ \\
\hline Central $^{b}$ & $\begin{array}{l}\text { The central decision point of the floor where the kitchen, } \\
\text { reception and waiting area is situated. }\end{array}$ & 2 \\
\hline Crossing ${ }^{b}$ & Simple crossings of hallways. & $1,3,4$ \\
\hline Startend $\mathrm{a}, \mathrm{b}$ & Decision points where the route starts or ends. & - \\
\hline
\end{tabular}

The results of the one-sided pairwise Mann-Whitney $U$ tests for the first categorization can be found in Table 5. It can be concluded that the symbol and text types (ST, T) are better suited to give 
a start or end instruction, while all other types got higher ratings at a decision point to take turns. This means that when the symbol and text type (ST) is considered as route instruction type, it is best used to start or end a route. For the overall highest rated picture + text type (PT), no significant differences were found, so this type is suited for every decision point category.

Table 5. Rankings of decision point categories 1 (level, startend, turn) from low to high ratings for every instruction type, resulting from pairwise one-tailed Mann-Whitney $U$ tests.

\begin{tabular}{c}
\hline Decision Point Categories 1 \\
\hline A \\
Startend $<$ turn \\
\hline AT \\
Level/ Startend $<$ turn \\
O \\
Startend $<$ Level $<$ turn \\
OT \\
Startend $<$ turn \\
M \\
Level Startend $<$ turn \\
MT \\
Level $<$ turn \\
P \\
Startend $<$ turn \\
ST \\
T \\
Level $<$ turn Startend \\
Level $<$ turn $<$ Startend \\
\hline
\end{tabular}

The analysis of the second categorization facilitates some more detailed conclusions (Table 6). The map and 3D types (M, MT, A, AT, O, OT) received significantly lower ratings at the stairs and at starting or ending points of routes compared to simple crossings, and for 3D additionally compared to the central decision point and the spiralstairs. This shows that the 3D simulations were more appreciated at complex decision points like the central decision point and the spiral stairs.

Table 6. Rankings of decision point categories 2 (stairs, elevator, spiralstairs, central, crossing) from low to high ratings for every instruction type, resulting from pairwise one-tailed Mann-Whitney $U$ tests.

\begin{tabular}{c}
\hline Decision Point Categories 2 \\
A \\
\hline Stairs/ Startend $<$ Crossing/ Central/ Elevator \\
AT \\
Stairs/ Startend $<$ Crossing \\
O \\
Stairs $<$ Central \\
OT \\
Startend $<$ Elevator/ Central/ Spiralstairs/ Crossing \\
Startend $<$ Central \\
M \\
MT \\
Stairs $<$ Crossing \\
P \\
Startend $<$ Crossing \\
ST \\
T \\
Stairs $<$ Startend/ Crossing/ Central \\
Stairs $<$ Startend/ Crossing/ Central \\
\hline
\end{tabular}




\subsubsection{Rankings of User Characteristics}

The previous method is repeated to analyze the differences between user characteristics. In Table 7 the rankings for age class are given and the differences between the answers to the general questions can be found in Table 8. Although some trends are visible in these rankings, fewer general conclusions can be made compared to the decision point categories. This implies that the environmental factors were more defining for the instruction type preference than the user characteristics for this case study.

Table 7. Rankings of age class from low to high ratings for every instruction type, resulting from pairwise one-tailed Mann-Whitney U tests.

\begin{tabular}{c}
\hline Age Class \\
\hline $\mathrm{A}$ \\
Old $<$ Middle $<$ Young \\
$\mathrm{AT}$ \\
Old $/$ Middle $<$ Young \\
$\mathrm{O}$ \\
Old $<$ Middle $/$ Young \\
$\mathrm{M}$ \\
Old $<$ Young \\
$\mathrm{P}$ \\
\hline Old $<$ Middle $/$ Young \\
\hline
\end{tabular}

Table 8. Rankings of user characteristics (answers to Q1, Q2, Q3 and Q4) from low to high ratings for every instruction type, resulting from pairwise one-tailed Mann-Whitney $U$ tests.

\begin{tabular}{cccc}
\hline (Q1) & (Q2) & (Q3) & (Q4) \\
\hline & $\mathrm{A}$ & & $\mathrm{A}$ \\
& $\mathrm{n}<\mathrm{y}$ & & $\mathrm{n}<\mathrm{y}$ \\
& & & $\mathrm{AT}$ \\
$\mathrm{NT}$ & $\mathrm{OT}$ & & $\mathrm{n}<\mathrm{y}$ \\
$\mathrm{n}<\mathrm{y}$ & $\mathrm{y}<\mathrm{n}$ & & $\mathrm{OT}$ \\
& & $\mathrm{M}$ & \\
& & $\mathrm{y}<\mathrm{n}$ & \\
& & $\mathrm{MT}$ & $\mathrm{MT}$ \\
& & $\mathrm{y}<\mathrm{n}$ & $\mathrm{n}<\mathrm{y}$ \\
& $\mathrm{P}$ & $\mathrm{P}$ & $\mathrm{P}$ \\
& $\mathrm{y}<\mathrm{n}$ & $\mathrm{y}<\mathrm{n}$ & $\mathrm{n}<\mathrm{y}$ \\
& $\mathrm{PT}$ & $\mathrm{PT}$ & $\mathrm{PT}$ \\
& $\mathrm{y}<\mathrm{n}$ & $\mathrm{y}<\mathrm{n}$ & $\mathrm{n}<\mathrm{y}$ \\
\hline
\end{tabular}

(Q1) Do you easily get lost in buildings? (Q2) Have you been to the iGent tower in Ghent, Belgium? (Q3) Do you often use navigation aids to find your way indoors? (Q4) Do you often use navigation aids to find your way outdoors?

Older people gave the lowest ratings and young people gave significantly higher ratings than middle and old aged people for the agent based 3D simulations (A, AT).

Participants who indicated they often get lost indoors (Q1) gave significantly higher ratings to the 3D simulation with overview + text type (OT) compared to participants with good wayfinding abilities. Participants who are familiar with the iGent tower $(\mathrm{Q} 2)$ liked the 3D simulation agent view type (A) more than first time visitors. For the picture types (P, PT) and 3D simulation overview (OT), this finding is reversed. Regular users of indoor wayfinding systems (Q3) disliked the map and photo types (M, MT, P, PT) more than non-regular users, while regular users of outdoor systems (Q4) liked three of these types together with most $3 \mathrm{D}$ types $(\mathrm{A}, \mathrm{AT}, \mathrm{OT})$ more compared to non-regular users. 


\section{Discussion}

\subsection{Symbol + Text (ST) and Text (T)}

From the results of the online survey, it can be concluded that participants gave the lowest ratings to these two types. However, previous research by Kray et al. (2003) [20] showed that the preference for instruction types does not necessarily coincide with the wayfinding performance. More sophisticated instruction types were preferred because these are interesting or fascinating. Analogously, in this study participants may have given the symbol and text types (ST, T) lower ratings because they are fairly simple, but that does not mean these types cannot be effective. In addition to the load on the user, the load on the system needs to be considered as well while selecting a route instruction type. This is especially the case for mobile devices as they have less working capacity than stationary displays [24]. As mentioned above, text and symbols require few resources for generation and display. Therefore, it could be beneficial for the performance of a wayfinding system to consider using these types. This study has shown that they are best used at start- and endpoints of indoor routes and are best avoided to change levels at stairs.

The non-significant difference in ratings between regular and non-regular users of wayfinding systems for ST and T could be an indication of the low cognitive demand of these types. The threshold to successfully interpret these simple instruction types could be lower than for other, more elaborate types, for which we did found significant differences between regular and non-regular users. Moreover, this study showed that these differences between regular and non-regular users are reversed for indoor and outdoor systems: non-regular users of indoor systems preferred photo and map types while non-regular users of outdoor systems did not prefer photo and map types. For the outdoor case, non-regular users of navigation aids are likely to use overview maps to find their way, because this was the standard wayfinding method before the booming of mobile navigation aids. As mentioned above, overview maps differ from the turn-by-turn instructions of wayfinding aids in many ways, e.g., they enable survey knowledge of an environment but in doing so also demand a high cognitive load of the wayfinder [3]. It is possible that participants who are used to outdoor wayfinding with overview maps had difficulties with the route knowledge provided by turn-by-turn instructions. These difficulties could have resulted in the lower ratings by non-regular users. To explain the reversed trend for indoor wayfinding systems, more information is needed on the alternatives used by non-regular users of wayfinding systems. As indoor wayfinding support is not yet common practice, no assumptions can be made on the use of these systems.

Another consistent trend in the ratings is the preference for instruction types with additional text, compared to the same types without text. A previous study of Beeharee \& Steed (2006) [24] showed that the reversed method, adding photos to text instructions, can enhance wayfinding performance. This indicates that combining multiple instruction types improves the usability of the system, because a combination of type-specific characteristics is facilitated that way. The text instruction demands little interpretation effort, imposing little cognitive load, but the images or 3D simulations are available for extra information if necessary. As stated in the introduction, the text instruction is easily combined with other types, but by doing so, the resources have to be increased.

\subsection{Photo (P and PT)}

It is clear from Table 3 that $\mathrm{P}$ and PT received the highest ratings. These findings support the idea that more detailed representations are preferred by wayfinders, in spite of the high cognitive load. However, participants who are familiar with the building gave lower ratings to the photo types than first-time visitors. This could be an indication that familiar users do not need a large amount of information and can even be disturbed by it. Besides a high cognitive load, another drawback of the photo types is the substantial workload to develop them. Moreover, every time changes are made to the building, new photos need to be captured in order to avoid confusing the users $[25,26]$. Augmented reality (AR), where information is projected on the real-life view, could solve this update 
issue. The design of the photo types in this research is compatible with AR route instructions: the symbol that indicates the required action is placed on the picture like it would be visualized with an AR system (Figure 3), resulting in so called augmented photographs. In recent years, AR has increasingly been considered as an alternative for route communication. However, research to date has been mostly restricted to the development of the technology and not to the usability of such a system [21]. This study confirms that AR is a promising route communication technique and that it would be useful to analyze the usability of AR in future research.

\subsection{Map (M and MT)}

The map types received lower ratings than the photo types. This result is consistent with the findings of other researchers $[12,20,21]$. Moreover, it confirms that, although maps may be suited to give a building overview, they should not be blindly adopted for turn-by-turn instructions. The narrow map area, which is depicted on the route instruction can impair orientation as distant landmarks are not visible [27]. This study might have shed some light on an additional reason why maps are not appreciated indoors, as the map type got particularly low ratings for the spiral stairs compared to other decision points. In modern buildings, creative architectural constructions to change floors, such as spiral stairs (decision point 8 in Figure 1), are increasingly common. Indoor maps have to face the challenge of visualizing these constructions, while this is less the case for outdoor maps [28,29]. This fundamental difference between the indoor and outdoor environment could explain the low ratings for maps as an indoor instruction type, while they are common practice for outdoor instructions.

\section{4. $3 D$-imulations ( $A, A T, O$ and $O T$ )}

The 3D and the photo types have similar characteristics (easy to recognize landmarks, high cognitive load), but 3D generally received lower ratings. A 3D simulation gives more information than an image, but also demands more interpretation time or cognitive load of the user [15]. The results of this survey indicate that participants generally preferred less information and less interpretation time, as the $3 \mathrm{D}$ types mostly received low ratings. However, at more complicated decision points (i.e., the central decision point and the spiral stairs), the 3D types scored better compared to other decision points. Moreover, people who answered yes to the question if they often get lost, gave higher ratings to the OT type than people who answered no. These findings could indicate that for a context that requires more information than usual, 3D simulations could help to ease the wayfinding decision.

Additionally, the preference for 3D depended on the age of the participants. Young people (age 18-29) gave higher ratings than middle aged people (age 29-49) to the agent based 3D simulations, while for the other types this effect was not significant. This shows that younger people can better interpret this type of 3D-instruction, while this is not necessarily the case for other types. Older people gave the lowest ratings to four types, indicating they have most difficulties interpreting route instructions. Finally, participants who are familiar with the iGent gave higher ratings to the agent-based view than non-familiar participants. This type of simulation is more easily translated to the environment when you have already visited the building, as it is filmed from the wayfinder's points of view.

\subsection{Limitations and Future Work}

Using an online survey as a research method has a lot of advantages, such as a large number of participants and controlled variables, but also some disadvantages. The first disadvantage is that often the duration of the survey has to be restricted to improve participation. To this end, in this survey the general questions were kept simple, which means that a limited amount of information is gained on the participant's characteristics. Additionally, only ten instruction types were shown, five of which were fundamentally different (e.g., photos compared to maps). Future research could focus on one specific type (e.g., photos) and analyze if changing some characteristics has an influence on the user preference or make different combinations of instruction types. Furthermore, the results of 
this research are promising for the use of augmented reality as a new route instruction type in indoor wayfinding support. Therefore, future research on the usability of this technology is essential.

A second disadvantage of online surveys is that participants are not able to move in the building itself. The limited screen size for example can have an effect on the results, although the route instructions in this survey had the same size as they would have on a mobile device [30,31]. Additionally, the effectiveness of the route instructions could not be tested with this setup. Therefore, the conclusions of this study will be validated with a real-life experiment where participants use a wayfinding system which is adapted according to the results of this study.

A final limitation is the case-study building for which the online survey was designed, as this test was not repeated for other buildings. Although the iGent tower has a common office building layout, it would be favorable to test if the building characteristics have an influence on the instruction type preference.

\section{Conclusions}

Adapted mobile wayfinding systems are being developed to facilitate intuitive indoor route guidance, by adapting the wayfinding information to the context. While navigating indoors with these systems, people are guided from decision point to decision point by turn-by-turn instructions. By adapting the type of these turn-by-turn instructions (map, photo, 3D, text, etc.), the right amount of information is given in the most suitable manner. To determine which type of instruction should be used in which context, an online survey was executed in which participants rated ten instruction types. Results show that the types that were combined with text scored overall better than the types without additional text. Of all types, the photo types received the best scores of unfamiliar users. The text and symbol types generally received the lowest ratings, but are best suited to start or end a route. Maps mostly got intermediate ratings, but were not appreciated at complex decision points. On these specific decision points, the 3D simulations gained preference. Additionally, some 3D simulations received higher ratings from younger people and participants with poor wayfinding abilities. The results of this study can be implemented in the design and development of mobile indoor wayfinding aids to facilitate more intuitive route guidance.

Author Contributions: Conceptualization, Methodology \& Formal analysis, L.D.C.; Writing-Review \& Editing, K.O., N.V.d.W. and N.V.; Supervision, P.D.M.

Funding: This research was funded by Research Foundation Flanders (FWO), grant number FWO17/ASP/242.

Conflicts of Interest: The authors declare no conflict of interest. The funders had no role in the design of the study; in the collection, analyses, or interpretation of data; in the writing of the manuscript, or in the decision to publish the results.

\section{References}

1. Reichenbacher, T. Adaptive methods for mobile cartography. In Proceedings of the 21th ICC, Durban, South Africa, 10-16 August 2003.

2. Gartner, G. Location-Based Mobile Pedestrian Navigation Services-the Role of Multimedia Cartography. In ICA UPIMap; Na: Tokyo, Japan, 2004; pp. 155-184.

3. Zimmer, H.D.; Baus, J.; Münzer, S.; Aslan, I.; Schwalm, M. Computer-assisted navigation and the acquisition of route and survey knowledge. J. Environ. Psychol. 2007, 26, 300-308. [CrossRef]

4. Puikkonen, A.; Sarjanoja, A.-H.; Haveri, M.; Huhtala, J.; Häkkilä, J. Towards designing better maps for indoor navigation-Experiences from a case study. In Proceedings of the 8th International Conference on Mobile and Ubiquitous Multimedia, Cambridge, UK, 22-25 November 2009.

5. Bouwer, A.; Nack, F.; El Ali, A. Lost in navigation: Evaluating a Mobile Map App for a Fair. In Proceedings of the 14th ACM International Conferences Multimodal Interact-ICMI 12 No, Santa Monica, CA, USA, 22-26 October 2012.

6. Schwering, A.; Krukar, J.; Li, R.; Anacta, V.J.; Fuest, S. Wayfinding through orientation. Spat. Cogn. Comput. 2017, 17, 273-303. [CrossRef] 
7. Allen, G.L. Principles and practices for communicating route knowledge. Appl. Cogn. Psychol. 2000, 14, 333-359. [CrossRef]

8. Giannopoulos, I.; Kiefer, P.; Raubal, M.; Richter, K.; Thrash, T. Wayfinding decision situations: A conceptualmodel and evaluation. In International Conference on Geographic Information Science; Springer: Cham, Germany, 2014; pp. 221-234.

9. Mackaness, W.; Bartie, P.; Sanchez-Rodilla Espeso, C. Understanding Information requirements in “Text Only" pedestrian wayfinding systems. In International Conference on Geographic Information Science; Springer: Cham, Germany, 2014; pp. 235-252.

10. Richter, K.-F.; Klippel, A. A Model for context-specific route directions. In Spatial Cognition IV; Freksa, C., Knauff, M., Bernd, K.-B., Nebel, B., Barkowsky, T., Eds.; Springer: Berlin, Germany, 2005; pp. 58-78.

11. Huang, H.; Gartner, G.; Schmidt, M.; Li, Y. Smart environment for ubiquitous indoor navigation. In Proceedings of the 2009 International Conference on New Trends in Information and Service Science, Beijing, China, 30 July 2009; pp. 176-180.

12. Chittaro, L.; Burigat, S. Augmenting audio messages with visual directions in mobile guides. In Proceedings of the 7th International Conference on Human Computer Interaction with Mobile Devices \& Services, Salzburg, Austria, 19-22 September 2005; pp. 107-114.

13. Mast, V.; Jian, C.; Zhekova, D. Elaborate descriptive information in indoor route instructions. In Proceedings of the 34th Annual Meeting of the Cognitive Science Society, Austin, TX, USA, 1-4 August 2012; pp. 1972-1977.

14. Fallah, N.; Apostolopoulos, I.; Bekris, K.; Folmer, E. Indoor human navigation systems: A survey. Interact. Comput. 2013, 25, 21-33. [CrossRef]

15. Ohm, C.; Müller, M.; Ludwig, B. Displaying landmarks and the user's surroundings in indoor pedestrian navigation systems. J. Ambient. Intell. Smart Environ. 2015, 7, 635-657. [CrossRef]

16. Lertlakkhanakul, J.; Li, Y.; Choi, J.; Bu, S. GongPath: Development of BIM Based Indoor Pedestrian Navigation System. In Proceedings of theNCM 2009 5th International Joint Conference INC, IMS, IDC, Seoul, Korea, 25-27 August 2009; pp. 382-388.

17. Ohm, C.; Müller, M.; Ludwig, B. Evaluating Indoor pedestrian navigation interfaces using mobile eye tracking. Spat. Cogn. Comput. 2017, 17, 89-120. [CrossRef]

18. Delikostidis, I.; Van Elzakker, C.P.J.M.; Kraak, M.J. Overcoming challenges in developing more usable pedestrian navigation systems. Cartogr. Geogr. Inf. Sci. 2016, 43, 189-207. [CrossRef]

19. Ooms, K.; Duytschaever, A.; Stroeken, K.; Verdoolaege, A.; Viaene, P.; van de Weghe, N. Fine-tuning the usability of a crowdsourced indoor navigation system. Cartogr. Geogr. Inf. Sci. 2018, 46, 456-473. [CrossRef]

20. Kray, C.; Elting, C.; Laakso, K.; Coors, V. Presenting route instructions on mobile devices. In Proceedings of the 8th international conference on Intelligent user interfaces, New York, NY, USA, 12-15 January 2003; pp. 117-124.

21. Walther-Franks, B.; Malaka, R. Evaluation of an augmented photograph-based pedestrian navigation system. In Proceedings of the 9th International Symposium on Smart Graphics, Rennes, France, 27-29 August 2008; pp. 94-105.

22. Alarifi, A.; Al-Salman, A.; Alsaleh, M.; Alnafessah, A.; Al-Hadhrami, S.; Al-Ammar, M.A.; Al-Khalifa, H.S. Ultra wideband indoor positioning technologies: Analysis and recent advances. Sensors 2016, 16, 707. [CrossRef] [PubMed]

23. Hart, A. Mann-whitney test is not just a test of medians: Differences in spread can be important. BMJ 2001, 323, 391-393. [CrossRef] [PubMed]

24. Butz, A.; Baus, J.; Krüger, A.; Lohse, M. A hybrid indoor navigation system. In Proceedings of the 6th international conference on Intelligent user interfaces, Santa Fe, NM, USA, 14-17 January 2004; pp. 25-32.

25. Beeharee, A.K.; Steed, A. A Natural wayfinding-Exploiting photos in pedestrian navigation systems. In Proceedings of the 8th conference on Human-computer interaction with mobile devices and services, Helsinki, Finland, 12-15 September 2006; pp. 81-88.

26. Kolbe, T.H. Augmented videos and panoramas for pedestrian navigation. Geowiss. Mitt. 2003, 66, 45-52.

27. Keil, J.; Mocnik, F.-B.; Edler, D.; Dickmann, F.; Kuchinke, L. Reduction of map information regulates visual attention without affecting route recognition performance. ISPRS Int. J. Geo Inf. 2018, 7, 469. [CrossRef]

28. Nossum, A.S. IndoorTubes a novel design for indoor maps. Cartogr. Geogr. Inf. Sci. 2011, 38, $192-200$. [CrossRef] 
29. Hölscher, C.; Meilinger, T.; Vrachliotis, G.; Brösamle, M.; Knauff, M. Up the down staircase: Wayfinding strategies in multi-level buildings. J. Environ. Psychol. 2006, 26, 284-299. [CrossRef]

30. Dillemuth, J.A. Navigation tasks with small-display maps: The sum of the parts does not equal the whole. Cartographica 2009, 44, 187-200. [CrossRef]

31. Willis, K.S.; Hölscher, C.; Wilbertz, G.; Li, C. A comparison of spatial knowledge acquisition with maps and mobile maps. Comput. Environ. Urban Syst. 2009, 33, 100-110. [CrossRef]

(c) (1)

(C) 2019 by the authors. Licensee MDPI, Basel, Switzerland. This article is an open access article distributed under the terms and conditions of the Creative Commons Attribution (CC BY) license (http://creativecommons.org/licenses/by/4.0/). 